\title{
RESPUESTA INADECUADA A LA VACUNA CONTRA LA HEPATITIS B EN PERSONAL DE SALUD DEL HOSPITAL NACIONAL, PARAGUAY.
}

\section{INAPPROPRIATE RESPONSE AGAINST HEPATITIS B VACCINE IN HEALTH PERSONNEL OF THE NATIONAL HOSPITAL, PARAGUAY.}

\author{
Raúl Emilio Real Delor ${ }^{1,4}$, Gustavo Villar², Rigel Espínola ${ }^{3}$.
}

\section{Resumen:}

Introducción: el personal de salud es un grupo de riesgo de adquirir la hepatitis B. La vacuna contra la misma es efectiva pero requiere evaluación de la respuesta inmune con el dosaje de antiHBs.

Objetivos: determinar la respuesta inmunológica a la vacuna contra la hepatitis B en médicos, enfermeras y estudiantes de Medicina del Hospital Nacional, Paraguay.

Métodos: se realizó un estudio observacional, analítico y transversal en una muestra de 120 trabajadores de la salud durante el 2017. Se determinó el nivel de antiHBs con inmunoensayo cuantitativo y se registraron variables demográficas y clínicas, previo consentimiento informado.

Resultados: la muestra se conformó con 79 mujeres y 41 varones. La edad media fue $28 \pm 7$ años. Se incluyeron médicos (62,5\%), enfermeras (20\%) y estudiantes de Medicina (17,5\%). El IMC medio fue $24,7 \pm 3,8 \mathrm{~kg} / \mathrm{m}^{2}$. Los niveles séricos adecuados de antiHBs (>100 UI/ $\mathrm{mL}$ ) se detectaron en $64 \%$ del personal de salud. La obesidad fue un factor asociado a mala respuesta a la vacuna ( $p 0,02)$. El sexo, la edad, el tiempo trascurrido desde la última dosis y el tabaquismo no resultaron factores significativamente asociados a la falta de respuesta a la vacuna.

Conclusión: la respuesta inadecuada a vacuna $\mathrm{HB}$ se halló en $36 \%$. La obesidad se asoció significativamente a la mala respuesta inmunológica.

Palabras clave: vacuna hepatitis B; antiHBs; personal de salud.

\section{Abstract:}

Introduction: health personnel are a risk group for acquiring hepatitis $\mathrm{B}$. The vaccine against it is effective but requires evaluation of the immune response with the dosage of anti-HBs.

Objectives: to determine the immunological response to the vaccine against hepatitis $B$ in physicians, nurses and medical students of the National Hospital, Paraguay.

Methods: an observational, analytical and cross-sectional study was conducted in a sample of 120 health workers during 2017. The level of anti-HBs was determined with quantitative immunoassay and demographic and clinical variables were recorded, with prior informed consent.

Results: the sample was integrated with 79 women and 41 men. The mean age was $28 \pm 7$ years. Physicians $(62.5 \%)$, nurses (20\%) and medical students (17.5\%) were included. The mean BMl was $24.7 \pm 3.8 \mathrm{~kg} / \mathrm{m}^{2}$. Adequate serum levels of anti-HBs (> $100 \mathrm{IU} / \mathrm{mL}$ ) were detected in $64 \%$ of the health personnel. Obesity was a factor associated with poor response to the vaccine ( $p$ 0.02). Sex, age, the time elapsed since the last dose and smoking were factors not significantly associated with the lack of response to the vaccine.

Conclusion: inadequate response to HB vaccine was found in $36 \%$. Obesity was significantly associated with poor immunological response.

Keywords: hepatitis B vaccine; anti-HBs; health personnel.

\footnotetext{
1 Dpto. de Medicina Interna. Hospital Nacional. Itauguá, Paraguay

2 Postgrado en Medicina Interna. Universidad Nacional de Itapúa, Paraguay

3 Dpto. de Laboratorio. Hospital Nacional. Itauguá, Paraguay

4 Email de contacto: raulemilioreal@gmail.com
} 


\section{Introducción}

La hepatitis $B(\mathrm{HB})$ afecta a más de dos billones de personas en todo el mundo, muchos de ellos con secuelas como la hepatitis crónica (20\%) o mueren por cirrosis o carcinoma hepatocelular $(0,03 \text { a } 0,06 \%)^{(1,2)}$. El Paraguay es un país de riesgo intermedio de HB crónica: $2-7 \%{ }^{(3)}$. La vacuna contra la HB es segura y efectiva contra más del $90 \%$ de los serotipos y genotipos del virus de la HB ${ }^{(2,4)}$. Está indicada en adultos pertenecientes a grupos de riesgo, entre ellos el personal de salud ${ }^{(5)}$.

La respuesta inmunogénica se produce en la mayoría de las personas vacunadas contra $\mathrm{HB}^{(2)}$. No obstante, existe 5 a $15 \%$ de no respondedores o con escasa respuesta, dejando a estos sujetos susceptibles de adquirir HB ${ }^{(6,7)}$. La detección del anticuerpo contra el antígeno de superficie del virus HB (antiHBs) es la técnica recomendada para evaluarla. Los sujetos vacunados se clasifican según su respuesta inmunogénica en no respondedor $(<9,9 \mathrm{UI} / \mathrm{mL})$, poco respondedor $(10-100 \mathrm{UI} / \mathrm{mL})$ y buen respondedor $(>100 \mathrm{UI} / \mathrm{mL})^{(8,9)}$. La causa de la falta de respuesta a la vacuna no está bien aclarada pero se ha demostrado que está ligado a genes del Complejo Mayor de Histocompatibilidad ${ }^{(9)}$. Aparte de este factor genético, se ha observado que la falta de respuesta a la vacuna se asocia a comorbilidades crónicas, la edad y el sexo ${ }^{(2,8,10)}$.

La medición de antiHBs está indicada en el personal de salud a los 30-90 días posteriores a la última dosis de la vacuna y sobre todo cuando no consta la fecha de la vacunación ${ }^{(9,11)}$. Conocer el estado inmune contra la HB ofrece más tranquilidad al personal pero no debe impedir el uso de medidas de protección universal dado que existen otras infecciones que pueden transmitirse por fluidos corporales ${ }^{(12)}$. La medición habitual post vacunal de antiHBs se demostró ser costo-efectiva y disminuye la utilización de gammaglobulina hiperinmune ${ }^{(13)}$. La exposición ocupacional a fluidos infectados genera mucho coste económico y gran tensión psicológica que podría evitarse en sujetos vacunados adecuadamente ${ }^{(14)}$. En caso de detectarse escaso o nulo nivel de anticuerpos protectores, existe una gran posibilidad que dosis de refuerzo contra la HB logren mejorar ese nivel de anticuerpos ${ }^{(15)}$.

El Hospital Nacional es un centro de atención de alta complejidad de la red de Salud Pública del Paraguay. Un estudio realizado en el mismo reveló que $47 \%$ de los hemodializados crónicos tiene mala respuesta a la vacuna contra $\mathrm{HB}^{(16)}$, generando alto riesgo de contagio al personal de salud en este centro asistencial, de ahí el interés de esta investigación.

Los objetivos fueron determinar el nivel de antiHBs en personal de salud del Hospital Nacional, describir sus características clínicas y demográficas, detectar los factores de riesgo asociados a la falta de respuesta inmunológica a la vacuna contra HB.

\section{Métodos}

Se realizó un estudio observacional, analítico y transversal en una muestra de 120 trabajadores de la salud. Se incluyeron varones y mujeres, mayores de 18 años de edad, que concurren regularmente al Hospital Nacional (Itauguá, Paraguay) durante el 2017.

Fueron excluidos los sujetos que nunca recibieron el esquema completo de la vacuna contra HB, los conocidos portadores de estados de inmunosupresión (congénita $\mathrm{O}$ adquirida), en quimioterapia $\mathrm{O}$ hemodiálisis crónica y aquellos con antecedente de haber padecido HB.

Se utilizó un muestreo por conveniencia de los que aceptaban participar del estudio previo consentimiento informado. Se midieron variables demográficas, clínicas, la fecha de última dosis de vacuna contra $\mathrm{HB}$ y el nivel de antiHBs. Este se efectuó mediante inmunoensayo cuantitativo de electroquimioluminiscencia (marca $\operatorname{COBAS}^{\circledR}$ ), en un equipo automatizado COBAS 6000, en el Servicio de Inmunología del Dpto. de Laboratorio del Hospital Nacional.

Las variables fueron analizadas con el programa Epi Info $7^{\circledR}$. Para determinar la respuesta inmune a la vacuna contra $\mathrm{HB}$, se reagrupó a los sujetos respondedores (antiHBs>100 Ul/mL) y no respondedores (antiHBs $<100 \mathrm{Ul} / \mathrm{mL}$ ). Se calculó el OR (IC 95\%) utilizando la prueba chi². Se consideró significativa toda $\mathrm{p}<0,05$.

El tamaño de muestra se calculó con el programa Epi Dat $3.1^{\circledR}$. En un universo de 1500 sujetos se esperó una prevalencia de $9 \%$ de no respondedores ${ }^{(6,7)}$. Para una precisión $5 \%$, nivel de confianza $95 \%$, el tamaño mínimo fue 117 sujetos.

\section{Aspectos éticos}


Se mantuvo el anonimato de los sujetos del estudio. No se hizo discriminación por género, raza, creencia religiosa o política. Los resultados del análisis de antiHBs fueron entregados personalmente a los participantes. A aquellos no respondedores a vacuna $\mathrm{HB}$, se les aconsejó recibir un esquema completo en el vacunatorio del Hospital Nacional y medición ulterior de antiHBs. Los análisis laboratoriales y las vacunas no tuvieron costo. Todos los sujetos contactados pudieron negarse a participar del estudio y no se sacó provecho de las personas dependientes. El protocolo de investigación fue aprobado por el Comité de Ética de la Facultad de Medicina de la Universidad Nacional de Itapúa. Los autores declaran que no existen conflictos de interés comercial.

\section{Resultados}

De los 120 trabajadores de la salud reclutados, 79 eran del sexo femenino (66\%) y 41 del sexo masculino (34\%). La edad media fue 28 \pm 7 años (rango 20-69 años). Se incluyeron médicos (41\%), estudiantes de Medicina (34\%) y enfermeras (25\%). El peso medio fue $70,4 \pm 11,7 \mathrm{~kg}$, la talla media $1,67 \pm 0,06 \mathrm{~m}$ y el IMC medio $24,6 \pm 3,3 \mathrm{~kg} / \mathrm{m}^{2}$.

Los encuestados refirieron haberse vacunado entre 1 y 21 años antes, siendo la mediana 3 años (rango intercuartílico 2-6 años). El nivel sérico medio de antiHBs fue $416 \pm 394 \mathrm{UI} / \mathrm{mL}$ (rango 2-1000 Ul/mL).

Aplicando punto de corte de antiHBs en $100 \mathrm{UI} / \mathrm{mL}$, se detectó $64 \%$ de buenos respondedores (figura 1).

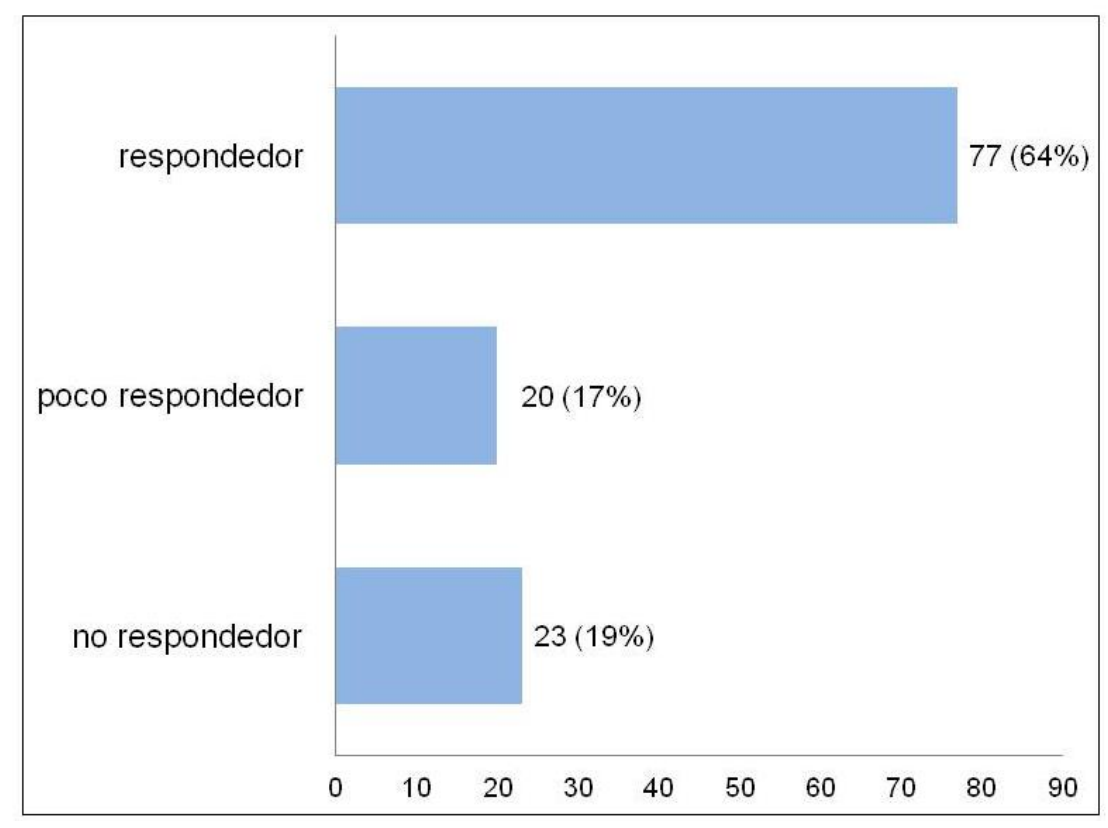

Figura 1. Estados de protección contra hepatitis B en personal de salud (n 120)

En el análisis multivariado se detectó que la obesidad fue un factor de riesgo significativo: OR 2,3 IC 95\% 1,1-5,1 ( $p$ 0,02), mientras que el tabaquismo y el sexo no lo fueron. Sólo hubo dos diabéticos: uno buen respondedor y otro sin respuesta inmune.

Se halló correlación negativa entre los niveles séricos de antiHBs y el tiempo transcurrido desde la vacunación $(r-0,2)$ y la edad $(r-0,05)$ aunque ambos no fueron significativos.

\section{Discusión}

La frecuencia de buena respuesta a vacuna contra HB detectada en nuestra muestra (64\%) es muy baja considerando que la literatura reporta 80 a $95 \%$ de respuesta adecuada ${ }^{(6,7)}$. Múltiples factores podrían explicar este fenómeno.

Es sabido que los niveles de antiHBs declinan con el tiempo: más del $60 \%$ de las personas que inicialmente tuvieron una buena respuesta antigénica presentarán declinación antigénica en los siguientes 15 años ${ }^{(9)}$. Varios estudios demuestran niveles $>10 \mathrm{UI} / \mathrm{mL}$ de antiHBs en $73 \%$ de sujetos entre 20 y 49 años, disminuyendo a $63 \%$ en aquellos mayores a 50 años ${ }^{(17,18)}$. Esta diferencia podría explicarse por los años 
que pasaron desde la última dosis de la vacuna ${ }^{(17,18)}$. Sin embargo, en nuestro estudio hubo sujetos con más de 10 años de vacunación y con niveles de antiHBs superiores a $100 \mathrm{Ul} / \mathrm{mL}$. No hallamos correlación entre los valores de antiHBs y edad $(r-0,05)$ ni con el tiempo trascurrido entre la última dosis $(r \quad 0,2)$. Suponemos que existen otros factores que impiden una adecuada respuesta inmune.

En relación al peso un estudio reporta que en personal de salud un índice de masa corporal (IMC) $<42 \mathrm{k} / \mathrm{m}^{2}$ se asocia a $3 \%$ de falta de respuesta, mientras que con $I M C \geq 42 \mathrm{k} / \mathrm{m}^{2}$ esa frecuencia aumenta a $14,9 \%{ }^{(19)}$. En nuestro reporte, la obesidad resultó un factor de riesgo significativo $(p 0,02)$ de mala respuesta a la vacuna. Esto coincide con el estudio de Young KM que demuestra un riesgo elevado de mala respuesta en obesos $(p 0,009)^{(20)}$. Ello se debe a que la obesidad produce un estado de inflamación crónica que incluye disregulación en la producción de citoquinas ${ }^{(20,21)}$.

El sexo masculino no resultó un factor de riesgo como se describe en varios reportes ${ }^{(9,10,21)}$. López $\mathrm{MH}^{(10)}$ detecta en el sexo masculino falta de respuesta en $25 \%$ vs $10 \%$ en las mujeres, mientras Mast EE ${ }^{(9)}$ halla el mismo fenómeno en $87 \%$ vs $12 \%$. Según estos autores, el motivo de la mala respuesta a la vacuna en los varones debería ser algún factor hormonal, aún no establecido.

Otros factores conocidos como riesgo de mala respuesta a la vacuna contra HB, como la diabetes mellitus y el tabaquismo, no fueron suficientemente frecuentes en nuestra muestra para poder ser analizados. Hay estudios que asocian niveles bajos de antiHBs en fumadores. Entre los no respondedores se reporta que $21,9 \%$ son fumadores, posiblemente por la disminución de las respuestas inmunes ${ }^{(21)}$. Se sabe que el tabaquismo reduce la respuesta antigénica de los linfocitos T helper $1^{(19)}$.

El acceso a la vacuna HB en personal de salud no es universal ${ }^{(9)}$. En la Argentina, en el personal de salud de Córdoba, la cobertura se registró en $67,5 \%{ }^{(22)}$. En los Estados Unidos, sólo $57,5 \%$ de los grupos de riesgo tienen la vacuna contra $\mathrm{HB}{ }^{(23)}$. Estudios realizados en Brasil indican que sólo $85 \%$ del personal de salud ha sido vacunado y $25 \%$ de ellos no completaron el esquema completo ${ }^{(24)}$. El personal sanitario y los estudiantes de las áreas de la salud no se realizan rutinariamente el dosaje de antiHBs post vacunal para determinar su estado de protección inmunológica ${ }^{(25)}$. Se han descrito muchas causas por las cuales los profesionales de la salud no están adecuadamente vacunados: recelo a los efectos colaterales, falta de percepción del riesgo de infección, ausencia de información sobre la HB, presión laboral, dificultades de acceso a la vacuna o costos de la misma ${ }^{(21)}$. En nuestra muestra, ninguno fue excluido por no haber recibido la vacuna HB. De hecho, una condición excluyente para ingresar a las carreras de la salud y a las residencias médicas del Paraguay es demostrar el esquema de vacunación completo con la cartilla oficial del Ministerio de Salud. La debilidad de nuestra investigación es que no se contó con dicha cartilla de vacunación a la vista de modo a verificar el esquema recibido, la vía de administración, las dosis recibidas y el tiempo exacto transcurrido hasta la fecha de este estudio (2).

Otro hallazgo interesante fue ninguno de los encuestados se había realizado con anterioridad su dosaje de antiHBs como está recomendado (11-13,26). Esto demuestra que, aún siendo personal de salud con conocimientos sobre la gravedad de la HB, requieren capacitación y control de sus superiores ${ }^{(21,27)}$. A partir de nuestro estudio, este aspecto sería considerado en el reglamento de acceso y permanencia del personal sanitario en el Hospital Nacional. Los sujetos sin seroconversión fueron invitados a recibir esquema completo de vacunación contra HB y serán monitoreados según un protocolo que se ha diseñado en base a estos hallazgos ${ }^{(10)}$

El personal de salud es un grupo de riesgo de adquirir la HB y la vacuna contra la misma ha demostrado su efectividad ${ }^{(28)}$. El acceso a la vacuna HB es un problema pendiente en Latinoamérica ${ }^{(29)}$. En el Paraguay, desde el 2002 esta vacuna forma parte del esquema de vacunación infantil, llegando en 2008 a cubrir el $80 \%$ de los lactantes ${ }^{(21)}$. Por dicho motivo, los actuales trabajadores de la salud dependen de su propia voluntad para vacunarse y controlar su seroconversión. La exposición anual mundial a HB de los trabajadores de la salud es elevada 5,9\% ${ }^{(21)}$. En países en vías de desarrollo, 40 a $60 \%$ de los casos de HB en este tipo de personal se debe a accidentes cortopunzantes ${ }^{(21)}$. De ahí la importancia de tomar medidas efectivas para asegurar la protección del personal sanitario ya que el nivel detectado en nuestro estudio ha sido insuficiente.

En conclusión, la respuesta inadecuada a vacuna HB se halló en $36 \%$ del personal de salud. La obesidad se asoció significativamente a la mala respuesta inmunológica. No se halló correlación entre el tiempo transcurrido entre la vacunación contra la HB, la edad y los niveles de antiHBs. 


\section{Bibliografía}

1. European Association for the Study of the Liver. EASL 2017 Clinical Practice Guidelines on the management of hepatitis B virus infection. J Hepatol. 2017 Aug;67(2):370-398. https://www.easl.eu/medias/cpg/management-ofhepatitis-B-virus-infection/English-report.pdf

2. Tajiri K, Shimizu Y. Unsolved problems and future perspectives of hepatitis B virus vaccination. World $J$ Gastroenterol. 2015 Jun 21;21(23):7074-83. https://www.ncbi.nlm.nih.gov/pmc/articles/PMC4476869/pdf/WJG-217074.pdf

3. Weinbaum CM, Mast EE, Ward JW. Recommendations for identification and public health management of persons with chronic hepatitis B virus infection. Hepatology. 2009 May;49(5 Suppl):S35-44. http://onlinelibrary.wiley.com/doi/10.1002/hep.22882/epdf

4. Pottie K, Greenaway C, Feightner J, Welch V, Swinkels H, Rashid M et al. Evidence-based clinical guidelines for immigrants and refugees. CMAJ. 2011 Sep 6;183(12):E824-925. https://www.ncbi.nlm.nih.gov/pmc/articles/PMC3168666/pdf/183e824.pdf

5. Thudi K, Yadav D, Sweeney K, Behari J. Physicians infrequently adhere to hepatitis vaccination guidelines for chronic liver disease. PLOS One. 2013 Jul 26;8(7):e71124. https://www.ncbi.n/m.nih.gov/pmc/articles/PMC3724808/pdf/pone.0071124.pdf

6. Roukens $A H$, Visser $L G$. Hepatitis $B$ vaccination strategy in vaccine low and non-responders: a matter of quantity of quality? Hum Vaccin. 2011 Jun;7(6):654-7.

http://www.tandfonline.com/doi/pdf/10.4161/hv.7.6.14986?needAccess=true

7. Pérez-López JA, García-Elorriaga G, Del Rey-Pineda G, Manjarrez-Téllez B. Antibodies against hepatitis $B$ after vaccination in health workers. Salud Publica Mex. 2011 May-Jun; 53(3): 205-6. http://www.scielosp.org/pdf/spm/v53n3/a03v53n3.pdf

8. Yao J, Qiu Y, Chen Y, Jiang Z, Shen L, Shan H, Dai X, Li Q, Liu Y, Ren W, Ren J. Optimal vaccination program for healthy adults in China. Hum Vaccin Immunother. 2015;11(10):2389-94. https://www.ncbi.nlm.nih.gov/pmc/articles/PMC4635859/pdf/khvi-11-10-1053674.pdf

9. Mast EE, Weinbaum CM, Fiore AE, Alter MJ, Bell BP, Finelli L, Rodewald LE, Douglas JM Jr, Janssen RS, Ward JW; Advisory Committee on Immunization Practices (ACIP) Centers for Disease Control and Prevention (CDC). A comprehensive immunization strategy to eliminate transmission of hepatitis $B$ virus infection in the United States: recommendations of the Advisory Committee on Immunization Practices (ACIP) Part II: immunization of adults. MMWR Recomm Rep. 2006 Dec 8;55(RR-16):1-33. https://www.cdc.gov/mmwr/preview/mmwrhtml/rr5516a1.htm

10. Lopes MH, Sartori AM, Souza TV, Mascheretti M, Chaves Tdo S. Hepatitis B revaccination for healthcare workers who are anti-HBs-negative after receiving a primary vaccination series. Rev Soc Bras Med Trop. 2012 Oct;45(5):639-42. http://www.scielo.br/pdf/rsbmt/v45n5/v45n5a18.pdf

11.Davison SA, Strasser SI. Ordering and interpreting hepatitis B serology. BMJ. 2014 Apr 17; 348: g2522. http://www.bmj.com/bmj/section-pdf/756691?path=/bmj/348/7957/Practice.full.pdf

12. Chen W, Gluud C. Vaccines for preventing hepatitis B in health-care workers. Cochrane Database Syst Rev. 2005 Oct 19;(4):CD000100. http://onlinelibrary.wiley.com/doi/10.1002/14651858.CD000100.pub3/epdf

13. Launay O, Floret D. Hepatitis B vaccination: a review. Med Sci (Paris). 2015 May;31(5):551-8. https://www.medecinesciences.org/articles/medsci/pdf/2015/06/medsci20153105p551.pdf

14.Zhang L, Liu J, Lu J, Yan B, Song L, Li L, Cui F, Zhang G, Wang F, Liang X, Xu A. Antibody response to revaccination among adult non-responders to primary Hepatitis $B$ vaccination in China. Hum Vaccin Immunother. 2015;11(11):2716-22. https://www.ncbi.n/m.nih.gov/pmc/articles/PMC4685679/pdf/khvi-11-11-1045172.pdf

15. Walayat S, Ahmed Z, Daniel Martin, Puli S, Cashman M, Dhillon S. Recent advances in vaccination of nonresponders to standard dose hepatitis B virus vaccine. World J Hepatol 2015; 7(24): 2503-2509. https://www.ncbi.nlm.nih.gov/pmc/articles/PMC4621464/pdf/WJH-7-2503.pdf

16. López N, Real Delor RE. Respuesta a la vacuna contra la hepatitis $B$ en pacientes en hemodiálisis crónica. Rev Virtual Soc Parag Med Int 2016;3(1):22-32. http://www.revista.spmi.org.py/index.php/rvspmi/article/view/46/288

17. McMahon BJ, Dentinger CM, Bruden D, Zanis C, Peters H, Hurlburt D, et al. Antibody levels and protection after hepatitis B vaccine: results of a 22-year follow-up study and response to a booster dose. J Infect Dis. 2009 Nov 1;200(9):1390-6. https://academic.oup.com/jid/article-lookup/doi/10.1086/606119

18. Spradling PR, Xing J, Williams R, Masunu-Faleafaga Y, Dulski T, Mahamud $A$ et al. Immunity to hepatitis $B$ virus (HBV) infection two decades after implementation of universal infant HBV vaccination: association of detectable residual antibodies and response to a single HBV challenge dose. Clin Vaccine Immunol. 2013 Apr; 20(4): 559-61. https://www.ncbi.nlm.nih.gov/pmc/articles/PMC3623410/pdf/zcd559.pdf

19. Nashibi R, Alavi SM, Yousefi F, Salmanzadeh S, Moogahi S, Ahmadi F, Farashahinejad M. Post-vaccination Immunity Against Hepatitis B Virus and Predictors for Non-responders Among Medical Staff. Jundishapur $J$ Microbiol. 2015 Mar 21;8(3):e19579. https://www.ncbi.nlm.nih.gov/pmc/articles/PMC4385250/pdf/jjm-08-0319579.pdf

20. Young KM, Gray CM, Bekker LG. Is obesity a risk factor for vaccine non-responsiveness? PLoS One. 2013 Dec 11;8(12):e82779. https://www.ncbi.nlm.nih.gov/pmc/articles/PMC3859613/pdf/pone.0082779.pdf

21. Ropero Álvarez AM, Pérez-Vilar S, Pacis-Tirso C, Contreras M, El Omeiri N, Ruiz-Matus C, Velandia-González M. Progress in vaccination towards hepatitis $B$ control and elimination in the Region of the Americas. BMC Public Health. $2017 \quad$ Apr https://www.ncbi.nlm.nih.gov/pmc/articles/PMC5392937/pdf/12889_2017_Article_4227.pdf 17;17(1):325. 
22. Acevedo G, Sanchez J, López L, Willington A, Burrone S, Farias A. Asociación de cobertura de vacunación con factores sociodemográficos, en trabajadores de centros de salud de primer nivel de atención, Córdoba-Argentina. Rev Fac Cien Med Univ Nac Cordoba. 2016;73(3):163-169. https://revistas.unc.edu.ar/index.php/med/article/view/14451/15369

23. Annunziata K, Rak A, Del Buono H, DiBonaventura M, Krishnarajah G. Vaccination rates among the general adult population and high-risk groups in the United States. PLoS One. 2012;7(11):e50553. https://www.ncbi.nlm.nih.gov/pmc/articles/PMC3511565/pdf/pone.0050553.pdf

24. Assunção AÁ, Araújo TM, Ribeiro RB, Oliveira SV. Hepatitis $B$ vaccination and occupation exposure in the healthcare sector in Belo Horizonte, Minas Gerais. Rev Saude Publica. 2012 Aug;46(4):665-73. http://www.scielo.br/pdf/rsp/v46n4/aop3554.pdf

25. Shokuhi Sh, Gachkar L, Alavi-Darazam I, Yuhanaee P, Sajadi M. Occupational Exposure to Blood and Body Fluids among Health Care Workers in Teaching Hospitals in Tehran, Iran. Iran Red Crescent Med J. 2012 Jul;14(7):4027. https://www.ncbi.nlm.nih.gov/pmc/articles/PMC3438432/pdf/ircmj-14-402.pdf

26. National Center for Immunization and Respiratory Diseases. General recommendations on immunization: recommendations of the Advisory Committee on Immunization Practices (ACIP). MMWR Recomm Rep. 2011 Jan 28;60(2):1-64. https://www.cdc.gov/mmwr/preview/mmwrhtml/rr6002a1.htm

27. Fainboim H, Marciano S, Di Benedetto N, Gadano A. Consenso argentino de hepatitis B. Acta Gastroenterol Latinoam. 2013 Mar;43(1):59-74. http://www.actagastro.org/numeros-anteriores/2013/Vol-43-N1/Vol43N1PDF18.pdf

28.Zheng YB, Gu YR, Zhang M, Wang K, Huang ZL, Lin CS, Gao ZL. Health care workers in Pearl River Delta Area of China are not vaccinated adequately against hepatitis B: a retrospective cohort study. BMC Infect Dis. $2015 \mathrm{Nov}$ 22;15:542. https://www.ncbi.nlm.nih.gov/pmc/articles/PMC4655081/pdf/12879_2015_Article_1278.pdf

29.Zampino R, Boemio A, Sagnelli C, Alessio L, Adinolfi LE, Sagnelli E, Coppola N. Hepatitis B virus burden in developing countries. World J Gastroenterol. $2015 \quad$ Nov 14;21(42):11941-53. https://www.ncbi.nlm.nih.gov/pmc/articles/PMC4641116/pdf/WJG-21-11941.pdf 